Radial and Nonradial Pulsations as Probes of Stellar Physics

ASP Conference Series, Vol. 259, 2002

C. Aerts, T.R. Bedding, \& J. Christensen-Dalsgaard, eds.

\title{
Pulsating Red Giants: New Results
}

\author{
J.R. Percy, A.G. Bakos, H. Dunlop, A. Hussain, L. Kassim,
} R. Kastrukoff, Z. Nyssa, J.B. Wilson

Department of Astronomy and Astrophysics, University of Toronto, Toronto ON Canada M5S 3H8; e-mail: jpercy@erin.utoronto.ca

\section{G.W. Henry}

Tennessee State University, Nashville TN 37203-3401; e-mail: henry@schwab.tsuniv.edu

\begin{abstract}
We have (1) accumulated long-term (up to 15 years) photoelectric photometry of 76 bright pulsating red giants (PRGs) and related stars, with $V$ amplitudes of 0.04 to $\geq 1$ magnitude; (2) used a variety of techniques, including autocorrelation, to determine both "short" radial pulsation periods, and long secondary periods; (3) determined pulsation modes using the $\mathrm{Q}$-value method; the modes range from fundamental to second overtone, independent of temperature; (4) tested wavelet analysis on small-amplitude PRGs; (5) made a special study of PRGs with $V$ amplitudes less than 0.1 magnitude; and (6) called further attention to the problem of the long secondary periods of these stars. Our studies provide a "model" for the analysis and interpretation of larger, fainter surveys such as MACHO and OGLE.
\end{abstract}

\section{Introduction}

Mira, the first pulsating red giant (PRG), was discovered over 400 years ago, and many more Mira and semi-regular variables were subsequently discovered. Stebbins \& Huffer (1930) published the first photoelectric survey of red giant variability; they found that detectable variability set in at about MOIII, and increased with decreasing temperature. In the 1970's, Eggen published a series of papers in which he surveyed and studied large numbers of PRGs, and classified them as small-, medium-, and large-amplitude red variables (SARVs, MARVs, LARVs). In the 1990's, several groups identified ultra-small-amplitude (0.01 to 0.1 magnitude) red variables (USARVs); in particular, the comprehensive survey of GK giants by Henry et al. (2000) extended the USARV phenomenon to as early as K2III. The present paper deals almost entirely with SARVs and USARVs.

In a recent paper, Wood (2000) states that "red giant stars are probably the least understood of all variable stars". Wood may be biased, but Art Cox, in his opening lecture at this colloquium, seemed to support this view, on the grounds that the structure, evolution, and pulsation of PRGs is dominated by convection - a process which is poorly understood in astrophysics. 


\section{Data and analysis}

Our data come from three sources: the American Association of Variable Star Observers (AAVSO) Photoelectric Photometry program, the AAVSO visual program (for a small section of this paper only), and the robotic telescopes of Tennessee State University. See Percy et al. (1996, 2001ab) for further details.

We use light curves, Fourier analysis, and a form of autocorrelation analysis, as complementary tools for period analysis. The first two of these are widely used and well understood, but autocorrelation is less so. It is useful for identifying characteristic time scales of variability, especially in stars which are not strictly periodic. It determines the cycle-to-cycle behaviour of a star, averaged over all the data. When used with Fourier analysis, it can be useful in choosing between the true period and alias periods, when the data have seasonal (or nightly) gaps. Burki et al. (1978) published a simple autocorrelation algorithm in which the data are shifted sideways in time, and the goodness of fit is determined. We have recently implemented this algorithm for the study of PRGs (Percy et al., 2001c).

In our own algorithm (Percy et al., 1993), we calculate $\Delta$ mag and $\Delta$ time for every pair of measurements, and plot the first (averaged in bins) against the second over an appropriate range of $\Delta$ time. Minima occur at multiples of the characteristic time scale $\tau$, and can be used to measure $\tau$. The persistence of the minima is a measure of the degree of periodicity. The height of the first maximum is a measure of the amplitude. The height of the minima depends on the average error in the measurements, and the degree of irregularity of the star. Our method cannot deal easily with complex multiperiodicity, though it can do so if there are two periods with a large ratio, which is often the case with PRGs. Our algorithm is similar to (but not as statistically elegant as) the "variogram" method of Eyer \& Genton (1999), but is not the same as "classical" autocorrelation analysis (Scargle, 1989).

\section{Results}

We have accumulated a large (76 stars) database of long-term (up to 15 years) photoelectric photometry of bright SARVs and USARVs, mostly M giants, but also a few class II and I stars, and chemically-peculiar stars. The first two dozen stars from the AAVSO Photoelectric Program were published by Percy et al. (1996). Another two dozen stars were published by Percy et al. (2001a). Long-term VRI photometry of 34 stars, obtained with a robotic telescope, was published by Percy et al. (2001b). In each case, periods and amplitudes were carefully determined by the three techniques mentioned above. The data are suitable for studying both the short-term and the long-term variability of PRGs; see Percy et al. (2001b) for 200-day and 5000-day light curves of 34 stars.

USARVs. In Percy et al. (2001d), we made a special study of $11 \mathrm{~K} 5$-M0 III stars (Henry et al., 2000) with amplitudes less than $0.1 \mathrm{mag}$. They have periods as short as 4.8 days, but their Q-values are similar to those of SARVs. Several also have multiple periods. We are presently analyzing an additional season of data on these stars, to investigate the consistency of their behaviour. Unlike Koen \& Laney (2000), we do not find periods less than 10 days in later-type M 
giants. We believe that the short periods in their paper are spurious, and are due to the strong aliasing characteristics of the HIPPARCOS epoch photometry.

Pulsation Modes. Percy \& Parkes (1998) determined pulsation modes through Q-values for a small set of SARVs which were studied by Percy et al. (1996); they found the stars to be pulsating in radial modes between the fundamental and the third overtone. We have now determined Q-values for $47 \mathrm{M}$ giants with well-determined "short" periods (some stars have two short periods): the radii are estimated from both spectral types and ( $V-K)$ colours; the masses are assumed to be $1.4 M_{\odot}$; the theoretical Q-values were taken from Ostlie \& Cox (1986) which should be adequate for these "warm" red giants. About half appear to pulsate in the fundamental, about a quarter in the first overtone, and about an eighth in the second overtone. The rest have Q-values larger than the fundamental; two are USARVs, one may be II-III class, one is W Boo if the period is 55 days (see below). There is no obvious correlation between pulsation mode and temperature.

Wavelet analysis can be used to track the changing amplitude and period of variable stars, and it has been used extensively on LARVs and MARVs (e.g. Szatmáry et al., 1994). We have used the wavelet software on the AAVSO web site (www.aavso.org, Foster, 1996). We have investigated its application to SARVs, especially to study possible amplitude variation and mode switching (Percy \& Kastrukoff, 2001). We first applied it to AAVSO photoelectric photometry and 5-day means of AAVSO visual photometry of the well-studied SARV EU Del, with a period of 62.5 days. We found that the method usually gave the 62.5-day period, but the "most significant period" occasionally switched to a one- or two-cycle/day alias. This seemed to occur during seasons of irregularity, in which the measurements (and the adjacent seasonal gaps) could be adequately fitted by the alias period. We also applied wavelet analysis to W Boo - a SARV which, according to Fourier analysis of AAVSO photoelectric photometry (Percy \& Desjardins, 1996) had switched between periods of 25, 35 and 50 days. The wavelet analysis confirmed that the most significant period clustered around values of $25 \pm 2,35 \pm 2$, and $55 \pm 2$ days. We conclude that wavelet analysis of SARVs can be effective, even if there are seasonal gaps, as long as it is used with care and caution, in conjunction with light curves, autocorrelation, and Fourier analysis.

Long Secondary Periods - whose cause is unknown - have been previously been found in LARVs and MARVs. We find them in at least half of our SARVs. The finite length of our datasets may bias against finding long periods in some stars. The ratio of the long period to the dominant radial period is remarkably constant - between 9 and 12 . This is consistent with the "parallel sequences" found by Wood (2000) in the period-luminosity diagram. The ratio does not appear to correlate with temperature. In EG And, the long period is exactly half the binary orbital period, so the long period appears to be ellipsoidal. However Hinkle (private communication) seems to rule out binarity as a general cause of this phenomenon. Cummings (1999), on the basis of a spectroscopic and photometric study of southern PRGs, suggests rotation as the most likely cause. Wood (2000), in a cogent review based on observations of LMC PRGs, rules out dust, non-radial pulsation, and rotation as a cause. He suggests that, if binarity is not the cause, then a new convectively induced oscillatory thermal 
mode must be invoked. Long secondary periods occur in other types of pulsating stars, such as RV Tauri stars and the SRd variable 89 Her.

\section{Future Plans}

In the near future, we plan to: (1) extend our database to other SARVs in the literature with well-determined periods; (2) use other means of radius determination (such as angular diameters), and semi-empirical masses, to improve our Q-values; (3) use wavelet analysis, and other techniques, to study the nature of the mode switching, amplitude variability, and irregularity of SARVs; (4) seek clues to the nature of the long secondary periods.

Acknowledgments. JRP thanks NSERC Canada, and GWH thanks NSF and NASA, for research support. We thank the AAVSO, especially Howard Landis and Janet Mattei, for providing access to data. JRP thanks his Toronto co-authors, who are students at the senior high school and undergraduate level; variable stars are ideally suited for student projects (Percy, 2000).

\section{References}

Burki, G., Maeder, A., \& Rufener, F. 1978, A\&A, 65, 363

Cummings, I. 1999, J. Astron. Data, 5, \#2

Eyer, L. \& Genton, M.G. 1999, A\&AS, 136, 421

Foster, G. 1996, AJ, 112, 1709

Henry, G.W., Fekel, F., Henry, S.M., \& Hall, D.S. 2000, ApJS, 130, 201

Koen, C. \& Laney, D. 2000, MNRAS, 311, 636

Ostlie, D.A. \& Cox, A.N. 1986, ApJ, 311, 864

Percy, J.R. 2000, in ASP Conf. Ser., Vol. 220, Amateur-Professional Partnerships in Astronomy, eds. J. Percy \& J. Wilson, (San Francisco ASP), 310

Percy, J.R. \& Desjardins, A. 1996, PASP, 108, 847

Percy, J.R. \& Kastrukoff, R. 2001, JAAVSO, submitted

Percy, J.R. \& Parkes, M. 1998, PASP, 110, 1431

Percy, J.R., Ralli, J., \& Sen, L.V. 1993, PASP, 105, 287

Percy, J.R., Desjardins, A., Yu, L., \& Landis, H.J. 1996, PASP, 108, 139

Percy, J.R., Dunlop, H., Kassim, L., \& Thompson, R.R. 2001a, IBVS, 5041

Percy, J.R., Wilson, J.B., \& Henry, G.W. 2001b, PASP, 113, 983

Percy, J.R., Hussain, A., Gomez-Forrellad, J.M., Garcia-Melendo, E. 2001c, IAU IBVS, submitted

Percy, J.R., Nyssa, Z., \& Henry, G.W. 2001d, IAU IBVS, submitted

Scargle, J.D. 1989, ApJ, 343, 874

Stebbins, J. \& Huffer, C.M. 1930, Publ. Washburn Obs., 15, 138

Szatmáry, K., Vinkó, J., \& Gál, J. 1994, A\&AS, 108, 377

Wood, P.R. 2000, PASA, 17, 18 